\title{
Predicting Factors for Successful Maturation of Autogenous Haemodialysis Fistulas After Salvage Percutaneous Transluminal Angioplasty in Diabetic Nephropathy: A Study on Follow-Up Doppler Ultrasonography
}

\author{
Eui-Yong Jeon, Young Kwon Cho, ${ }^{2, *}$ Sung Bum Cho, Dae Young Yoon, ${ }^{2}$ and Seong O Suh ${ }^{4}$ \\ ${ }_{2}^{1}$ Department of Radiology, Sheikh Khalifa Specialty Hospital, Ras Al Khaimah, United Arab Emirates \\ ${ }_{3}^{2}$ Department of Radiology, Kangdong Seong-Sim Hospital, Hallym University College of Medicine, Seoul, Republic of Korea \\ ${ }_{4}^{3}$ Department of Radiology, Korea University Anam Hospital , Korea University College of Medicine, Seoul, Republic of Korea \\ ${ }^{4}$ Department of Internal Medicine, National Police Hospital, Seoul, Republic of Korea \\ ${ }^{*}$ Corresponding author: Young Kwon Cho, Department of Radiology, Kangdong Seong-Sim Hospital, Hallym University College of Medicine, 150, Seongan-ro Gangdong-Gu, Seoul, \\ 134701, Republic of Korea. Tel: +82-222242312, Fax: +82-222242481, E-mail: ykchoman@naver.com
}

Received 2015 August 19; Revised 2015 September 24; Accepted 2015 October 7.

\begin{abstract}
Background:Maturation failure of autogenous arteriovenous fistula (aAVF) has been increasing after surgical procedures and the salvage percutaneous transluminal angioplasty (SPTA) for immature aAVF has been identified as an effective treatment modality.

Objectives: The aim of this study was to identify factors predicting successful aAVF maturation and to determine positive technical aspects of sPTA.

Patients and Methods: We retrospectively reviewed medical records and radiological images of 59 patients who had undergone sPTA for non-maturing aAVFs. We analysed images from pre-surgical mapping Doppler ultrasonography, angiography, and angioplasty and follow-up Doppler ultrasonography performed within two weeks after sPTA. We assessed the following factors, for their ability to predict successful aAVF maturation:1) patient factors (age; sex; co-morbidities; and aAVFage, side and type);2) vessel factors (cephalic vein diameter and depth, presence of accessory veins, and pre- and postoperative radial artery disease);3) lesion factors (stenosis number, location and severity); and 4) technical factors (presence of residual stenosis and anatomic success ratio (ASR) on follow-up Doppler ultrasonography). Results: The technical and clinical success rates were both 94.9\%(56/59); the mean ASR was 0.84. An ASR of $\geq 0.7$ and no significant residual stenosis (<30\%) (both $\mathrm{P}<0.001$ ) on two-week follow-up Doppler ultrasonography predicted successful aAVF maturation.

Conclusion: For more precise prediction of successful aAVF maturation after sPTA, short-term follow-up Doppler ultrasonography $(<2$ weeks) was useful. If the ASR was $<0.7$ or if residual stenosis was $\geq 30 \%$, immediate repeat sPTA is recommended.
\end{abstract}

Keywords: Arteriovenous Fistula, Autologous Transplantation, Doppler Ultrasonography, Angioplasty

\section{Background}

Autogenous arteriovenous fistula (aAVF) for haemodialysis access is associated with a lower incidence of stenosis, occlusion and other postoperative complications. It has a mean patency period of 58-70 months, compared to the mean patency period of 24 - 36 months for synthetic grafts (1). Therefore, the Dialysis Outcomes Quality Initiative Guidelines recommend the use of aAVFs as the first choice for vascular access, despite the disadvantages of a longer maturation time and high failure rate of maturation (up to 50\%) compared to those for synthetic grafts (1, 2). To reduce failed aAVF maturity, Doppler ultrasonography (US) and/or venography has been used to screen for stenosis of the venous limb or for accessory veins, which are the most common causes of aAVF failure $(3,4)$.

Many studies have reported that percutaneous transluminal angioplasty (PTA) is a well-known and effective modality for salvaging immature $\operatorname{aAVFs}(5,6)$. However, technical and clinical factors that can predict successful
aAVF maturation after salvage PTA (sPTA) have not been well elucidated, especially with respect to the role of Doppler US during and after SPTA $(5,6)$.

\section{Objectives}

The purpose of this study was to identify factors predicting successful aAVF maturation during and after sPTA by means of Doppler US measurement.

\section{Patients and Methods}

\subsection{Patients}

The local institutional review board approved this study, and the requirement for informed consent was waived because of the retrospective nature of the study.

Fifty-nine patients who had undergone sPTA for immature aAVFs were included in this study. They were selected

Copyright ( 2016, Tehran University of Medical Sciences and Iranian Society of Radiology. This is an open-access article distributed under the terms of the Creative Commons Attribution-NonCommercial 4.0 International License (http://creativecommons.org/licenses/by-nc/4.0/) which permits copy and redistribute the material just in noncommercial usages, provided the original work is properly cited. 
from 478 patients with end-stage renal disease who had undergone aAVF creation for haemodialysis access from November 2008 to May 2014 (primarily during the latter three years). Two board-certified vascular surgeons with $>10$ years of experience created the aAVFs during the study period.

The subjects included 32 males and 27 females, and their ages ranged from 40 to 85 years, with a mean age of 66 years (Table 1). The presence of hypertension, diabetes mellitus, cardiovascular disease, and/or end-stage renal disease was evaluated to determine risk factors. If a patient had more than two risk factors, he or she was considered to have co-morbidity.

Thirty-nine patients had undergone haemodialysis via permanent haemodialysis catheters for a period ranging from two days to 4.5 years (mean, 154 days) before AVF creation. Nine patients were to undergo a second operation due to primary AVF maturation failure.

A total of 51 patients underwent radiocephalic (RC) AVF creation, and the remaining eight patients underwent brachiocephalic (BC) AVF creation. The fistula age, defined as the time period between the AVF surgery and SPTA, ranged from 18 to 152 days (mean, 92.8 days). Fiftyfive patients had co-morbidities (diabetic nephropathy in 59 and hypertension in 55).

All Doppler US examinations and PTA procedures were performed by two board-certified interventional radiolo- gists with $>15$ years of experience in vascular interventions. We did not include patients with recurring stenosis of the aAVF after initial clinical success in this series.

\subsection{Doppler Ultrasonography}

Routine preoperative mapping Doppler US was performed to assess the status of the cephalic veins on both arms from the wrist joint to the elbow in longitudinal and transverse directions. The following characteristics were recorded: 1) size of the cephalic vein, 2) tortuosity of the cephalic vein, 3) depth of the cephalic vein, 4) presence of accessory veins, 5) stenosis of the cephalic vein, and 6) stenosis or significant calcification of the radial artery. All Doppler US procedures were performed with IU-22 and HDI-5000 US machines (Philips Ultrasound, Bothell, WA, USA) using a 5 - $10 \mathrm{MHz}$ linear transducer.

The criteria for favourable pre-surgical AVF creation were: 1 ) size of the cephalic vein being $>2 \mathrm{~mm}$ in the forearm and $>2.5 \mathrm{~mm}$ in the arm, 2) no evidence of significant stenosis or thrombi of the cephalic vein, 3 ) no visible ipsilateral central vein stenosis or occlusion, and 4) size of radial artery being $>1.5-2 \mathrm{~mm}$ (4).

The criteria for suspicious aAVF maturation failure were: 1) weak or no thrill on physical examination, and 2) size of $<4 \mathrm{~mm}$ or volume flow of $<500 \mathrm{~mL} /$ minute within four weeks after the surgery (7).

Table 1. Analysis of Patient Factors That May Predict Successful Maturation of Autogenous Arteriovenous Fistula After Salvage Percutaneous Transluminal Angioplasty ${ }^{\mathrm{a}}$

\begin{tabular}{|c|c|c|c|c|}
\hline Patient Characteristics & Number of Fistulas $(\mathrm{N}=59)$ & Failed Maturation of AVF & Successful Maturation of AVF & P Value \\
\hline Gender & & & & 0.243 \\
\hline Male & 32 & 3 & 29 & \\
\hline Female & 27 & 0 & 27 & \\
\hline Patient age & & & & 0.219 \\
\hline$<65$ years & 37 & 1 & 36 & \\
\hline$\geq 65$ years & 22 & 2 & 20 & \\
\hline Co-morbidities & & & & 0.284 \\
\hline Present & 55 & 2 & 53 & \\
\hline Absent & 4 & 1 & 3 & \\
\hline Preop. vein diameter & & & & 0.583 \\
\hline$<2.5 \mathrm{~mm}$ & 31 & 2 & 29 & \\
\hline$\geq 2.5 \mathrm{~mm}$ & 28 & 1 & 27 & \\
\hline Preop. vein depth & & & & 0.586 \\
\hline$<3 \mathrm{~mm}$ & 28 & 2 & 26 & \\
\hline$\geq 3 \mathrm{~mm}$ & 31 & 1 & 30 & \\
\hline Type of fistula & & & & 0.508 \\
\hline RC-AVF & 51 & 3 & 48 & \\
\hline BC-AVF & 8 & 0 & 8 & \\
\hline Side of fistula & & & & 0.546 \\
\hline Right & 7 & 0 & 7 & \\
\hline Left & 52 & 3 & 49 & \\
\hline
\end{tabular}

\footnotetext{
${ }^{\mathrm{a}}$ Abbreviations: AVF, arteriovenous fistula; BC-AVF, Brachiocephalic arteriovenous fistula; Preop., preoperation; RC-AVF, radiocephalic arteriovenous
} fistula. 
Doppler US for detection of immature aAVFs was performed in three steps: 1 ) standard measurements of the diameter of the overall AVF draining vein, 2) visual estimation of the size at the focal stenotic or diffuse narrowed portions of the vein, which most severely limit venous flow on the draining vein of the AVF, respectively, and 3) volume flow measurements at the normal sized draining vein of the AVF. Measurement of volume flow at the focal stenotic or narrowed portions was avoided due to turbulent or laminar flow.

All patients with suspected aAVF maturation failure underwent Doppler US examination (in most cases by the same interventional radiologist who performed the preoperative evaluation) after surgical fistula creation, to evaluate changes in size, depth, course of the cephalic vein, as well as the presence of intraluminal stenosis in the cephalic vein and accessory veins. During follow-up Doppler US examination, we used several methods in an attempt to measure the identical lesion location of the cephalic vein (such as comparing the current US image to previous Doppler US images, with the same examiner who performed the previous Doppler US examination, and obtaining consensus between two interventional radiologists).

\subsection{Salvage Percutaneous Transluminal Angioplasty}

Arterial access through the brachial $(n=50)$ or radial artery $(n=9)$ was originally utilized to reduce the risk of injury to the immature distal trunk of the fistula during puncture or haemostasis after sPTA, especially when the distal trunk of the fistula was $<2 \mathrm{~mm}$ on US examination. Puncture was routinely performed with a 21-G Micropuncture set $®$ (Cook, Bloomington, IN, USA), under ultrasonographic guidance. The brachial artery was punctured in the antegrade direction for RC-AVF and in the retrograde direction for BC-AVF. After insertion of a 4 Fr short vascular sheath (Terumo, Tokyo, Japan), angiography was performed to detect stenosis, thrombosis, and accessory veins in the entire trunk of the fistula, arteriovenous anastomosis, artery and veins.

After the passage of a 0.014 or 0.018 inch micro-guide wire, a 2.5 - $5.0 \mathrm{~mm}$ balloon catheter (Powercross TM; Covidien Corp., Plymouth, MN, USA) was advanced and inflated up to $14 \mathrm{mmHg}$ at the site of the stenosis for one to five minutes after routine injection of 3,000 - 5,000 IU of heparin (8). The strategy for selecting balloon size was to select one with the same diameter or one $0.5 \mathrm{~mm}$ in diameter smaller than the diameter of the normal portion of the cephalic vein.

If a residual stenosis of $>30 \%$ remained after the initial PTA with the conventional balloon catheter, additional angioplasty was carried out with a same-sized, high-pressure balloon (Conquest $®$; Bard, Tampe, AZ, USA) or a cutting balloon (FlextomeTM; Boston Scientific, Natick, MA, USA). If there was difficulty in crossing the anastomosis with the above-described balloon catheter due to its stiff nature and large diameter, we performed an additional puncture of the radial artery distal to the anastomosis under ultra- sonographic guidance. The endpoint of the sPTA was: 1) residual stenosis of $<30 \%, 2$ ) no visible dissection, and 3) improved luminal flow on completion angiography $(9,10)$. All sPTA procedures were performed by two board-certified interventional radiologists with $>15$ years of experience in vascular interventions. Haemostasis of the arterial puncture site was achieved by using a haemostatic closure pad (Clo-Surplus PADTM; SCION, Miami, FL, USA) and haemostatic compression bandage (TR BandTM; Terumo, Tokyo, Japan). The interventional radiologists who performed the sPTAs confirmed complete haemostasis of the puncture site for all cases.

The results of the sPTA were evaluated in accordance with the standards of the society of vascular surgery, the American association for vascular surgery, and the society of interventional radiology $(9,10)$. If luminal flow through the entire fistula trunk to the central vein was completely restored and the extent of residual stenosis remained below $20 \%$ of the initial value, on completion of the angiography, the procedure was considered a technical success. If sufficient vascular maturation occurred and haemodialysis was successfully performed with a mean flow rate of $300 \mathrm{~mL} / \mathrm{minute}$ at least three times, the procedure was considered a clinical success (11). Primary patency was defined as the time period for which the aAVF maintained a sufficient flow rate for haemodialysis without revision after the first PTA. Secondary patency was defined as the time period between the first PTA and surgical revision or abandonment (12).

The anatomic success ratio (ASR) was calculated by dividing the diameter of the dilated segment of the venous limb of the fistula checked by follow-up US two weeks after SPTA, by the diameter of the dilated segment of the venous limb of the fistula checked by US immediately after sPTA. For ASR measurement, a Doppler US examination was performed immediately after SPTA, before haemostasis of the puncture site, and within two weeks after sPTA for all patients. In addition, the diameter of the non-stenotic venous limb of the AVF was measured again as a normal diameter, and the lumen of the venous limb of the fistula was examined for the presence of a thrombus or dissection.

If the ASR was $<0.7$ and physical examination did not reveal a distinct thrill, additional secondary Doppler US was performed in the next two weeks. If the ASR decreased further and the thrill was more aggravated on the second follow-up US compared to that of the first follow-up US, additional sPTA was performed immediately in the same manner as for the first sPTA.

\subsection{Statistical Analysis}

We analysed the data from all medical records and radiological images, including those obtained during preoperative mapping and postoperative Doppler US, SPTA, and follow-up Doppler US after sPTA.

The definition of maturation success was as follows: 1) a main trunk diameter of the fistula of $>4 \mathrm{~mm}$, and 2) a volumeflow through the main trunk of the fistula of $\geq 300 \mathrm{~mL} /$ 
minute. The definition of maturation failure was anything in contrary to the definition of maturation success (13).

The following factors were analysed with Cox univariate and multivariate analyses for their ability to predict successful maturation of aAVFs after sPTA: 1 ) patient factors (age $[<65$ years or $\geq 65$ years], gender [male or female], presence of co-morbidities, such as diabetes and hypertension [present or absent], age of the aAVF $[<60$ days or $\geq 60$ days], side of aAVF [right or left], and type of aAVF [RC or BC]);2) vessel factors (diameter of the cephalic vein on preoperative imaging $[<2.5 \mathrm{~mm}$ or $\geq 2.5 \mathrm{~mm}]$, depth of the cephalic vein on preoperative imaging $[<3.0 \mathrm{~mm}$ or $\geq 3.0 \mathrm{~mm}$ ], accessory veins [present or absent], and radial artery disease [present or absent]);3) lesion factors (type of lesion [stenosis or occlusion], number of lesions [single or multiple], location of the lesion [juxta-anastomotic or $>2 \mathrm{~cm}$ from anastomosis], and severity of stenosis [50\% - 74\%, 75\% - 89\%, 90\% - 99\%]); and 4) technical sPTA factors (residual stenosis [present or absent] and ASR on follow-up Doppler US $[<0.7$ or $\geq 0.7]$ ).

Vascular lesions that led to aAVF maturation failure were classified as follows:1) stenosis or 2) occlusion without thrombosis, based on the findings of postoperative Doppler US examination and SPTA. Stenosis was defined as a $\geq 50 \%$ reduction in the luminal diameter. A thrombus was defined as an echogenic mass of blood clots within the vascular lumen on Doppler US and a filling defect within the lumen surrounded by contrast material on sPTA (8). A significant accessory vein was defined as a venous channel originating from the main trunk of the fistula, similar in size to the main trunk, and reducing the flow through the main trunk of the fistula (9).

The location of the lesion was classified as:1) native radial artery, adjacent to the anastomosis; 2 ) arteriovenous anastomosis; 3 ) juxta-anastomotic vein (in the fistula within 2 $\mathrm{cm}$ from anastomosis); 4 ) venous outflow ( $>2 \mathrm{~cm}$ from the anastomosis, cannulation zone); 5) distal outflow (defined as a draining portion of the cephalic vein above the elbow joint for RC-AVF and above the mid-humerus for BC-AVF); and 6) central venous system (14). The diameter of the stenotic lesion was measured at the narrowest segment in the trunk of the fistula, and the normal diameter was defined as the largest diameter of the fistula.

Comparisons between groups were performed with the chi-square or Fisher's exact test for categorical variables. The risk factors against and predictors for successful fistula maturation were analysed with Cox multivariate analysis. Technical factors of the sPTA were analysed with the MannWhitney U-test. Primary and secondary patency after sPTA was analysed with the Kaplan-Meier method. The SPSS software version 19.0 for Windows (SPSS; IBM Corp., Chicago, IL, USA) was used for statistical analysis, and P values of $<$ 0.05 were considered statistically significant.

\section{Results}

The results of the detailed analysis of the vessel and lesion characteristics obtained during SPTA and pre- and postoperative Doppler US (Figure 1) are shown in Tables 2 and 3. All 59 patients underwent additional follow-up Doppler US between 10 days and two weeks after SPTA. Two patients had an ASR of $<0.7$ on the first and second follow-up Doppler US, and one patient was converted to open repair.

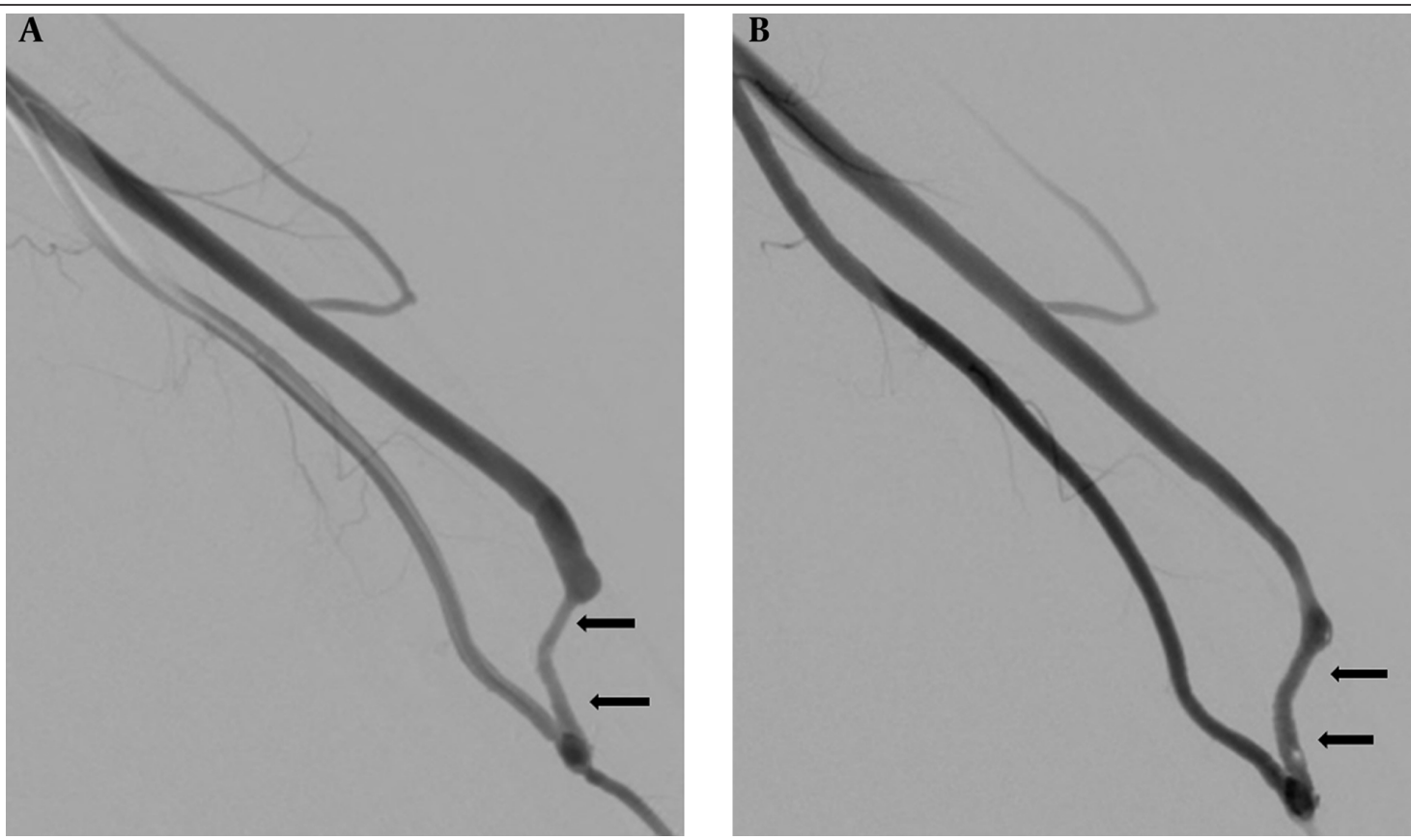

Figure 1. Successful fistula maturation after salvage PTA in a 54-year-old male with a non-maturing autogenous radiocephalic AVF; A, Initial angiography shows a focal severe stenosis (arrows) in the juxta-anastomotic vein of AVF; B, Angiography performed after the salvage PTA shows overall improvement of the stenosis, with subtle residual lesions (arrows) in the juxta-anastomotic vein of the AVF. The anatomic success ratio was measured with Doppler ultrasonography two weeks after the first salvage PTA was 0.8 (not shown). 
Table 2. Vessel Factors That Changed After Salvage Percutaneous Transluminal Angioplasty ${ }^{\mathrm{a}}$

\begin{tabular}{|c|c|c|}
\hline & Preop. & Postop. \\
\hline \multicolumn{3}{|c|}{ Mean diameter of cephalic vein $(\mathrm{cm})$} \\
\hline RC-AVF & 0.228 & 0.411 \\
\hline BC-AVF & 0.232 & 0.413 \\
\hline \multicolumn{3}{|c|}{ Mean depth of cephalic vein $(\mathrm{cm})$} \\
\hline RC-AVF & 0.267 & 0.299 \\
\hline BC-AVF & 0.272 & 0.278 \\
\hline \multicolumn{3}{|c|}{ Presence of fistula stenosis } \\
\hline RC-AVF & 3 & 51 \\
\hline BC-AVF & 2 & 8 \\
\hline \multicolumn{3}{|c|}{ Presence of accessory vein } \\
\hline RC-AVF & 4 & 6 \\
\hline BC-AVF & 0 & 0 \\
\hline \multicolumn{3}{|c|}{ Presence of arterial stenosis } \\
\hline Radial artery & 4 & 0 \\
\hline Brachial artery & 0 & 0 \\
\hline
\end{tabular}

a Abbreviations: AVF, arteriovenous fistula; BC-AVF, Brachiocephalic arteriovenous fistula; Postop., postoperative; Preop., preoperative; RCAVF, radiocephalic arteriovenous fistula.

Table 3. Lesion Factors for Immature Autogenous Arteriovenous Fistula Before Salvage Percutaneous Transluminal Angioplasty ${ }^{\mathrm{a}}$

\begin{tabular}{lcc}
\hline & RC-AVF $(\mathbf{N}=\mathbf{5 1})$ & BC-AVF $(\mathbf{N}=\mathbf{8})$ \\
\hline Degree of stenosis $^{\mathbf{b}}$ & & \\
$50-74$ & 24 & 5 \\
$75-89$ & 16 & 1 \\
$90-99$ & 11 & 2 \\
Stenosis location $\mathbf{b}$ & & 0 \\
Anastomosis & 3 & 2 \\
\hline Juxta-anastomotic vein & 45 & 4 \\
2 -10 cm of fistula & 1 & 2 \\
\hline Multiple & 2 & \\
Fistula diameter & & \\
Normal (mean) & $2.16-6.53(4.72)$ & $4.8-7.95(5.66)$ \\
Stenosis (mean) & $0.5-2.9(1.36)$ & $0.5-2.36(1.56)$ \\
\hline
\end{tabular}

a Abbreviations: BC-AVF, Brachiocephalic arteriovenous fistula; RC-AVF, radiocephalic arteriovenous fistula.

$\mathrm{b}_{\text {Values are presented as number. }}$

The first sPTA was technically and clinically successful in 56 and 54 patients, respectively. Furthermore, the second sPTA was clinically successful in two cases, in spite of the technical success of the first sPTA. Therefore, the overall technical and clinical success rates were both $94.9 \%$ (56/59). Neither technical nor clinical success was achieved in three patients after one to three sPTA procedures (Figure 2). There were no complications related to the SPTA procedure, such as hematoma or infection, except for one case of pseudoaneurysm after sPTA of an RC-AVF with a samesized balloon catheter for stenosis of the juxta-anastomotic vein, and in which was considered a technical failure of the first sPTA. Consequently, an additional BC-AVF was created in the ipsilateral arm (Figure 3). The mean ASR on follow-up Doppler US after sPTA was 0.84 (range, 0.28 -1.23), and the mean ASRs in the RC-AVF and BC-AVF groups
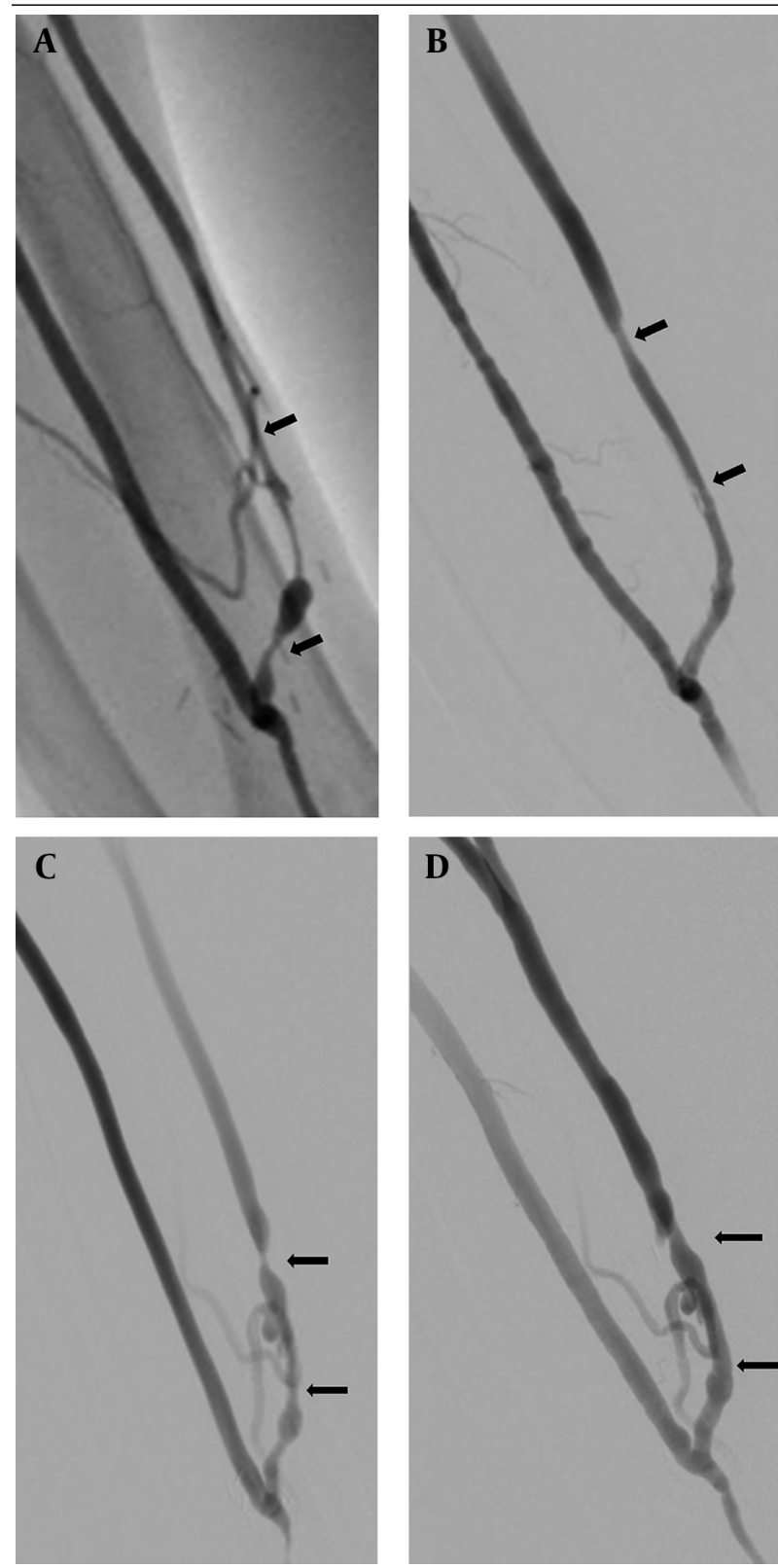

Figure 2. Failed fistula maturation after two sessions of salvage PTAs in a 72-year-old male with a non-maturing autogenous radiocephalic AVF; A, Initial angiography showed a focal severe stenosis (arrows) in the juxta-anastomotic vein of the AVF; B, Angiography performed after the first salvage PTA showed improvement of the stenosis with subtle residual lesions (arrows) in the juxta-anastomotic vein of the AVF. The anatomic success ratio measured with Doppler ultrasonography two weeks after the first salvage PTA was 0.5 (not shown). C, A second angiography performed four weeks after the first salvage PTA revealed the recurrence of severe multifocal stenosis (arrows) in the juxta-anastomotic vein of the AVF and weak accessory veins. D, Angiography after the second salvage PTA showed significant improvement of the stenosis with subtle residual lesions (arrows) in the juxtaanastomotic vein of the AVF. The anatomic success ratio measured with Doppler ultrasonography two weeks after the second salvage PTA was 0.4 (not shown). AVF, arteriovenous fistula; PTA, percutaneous transluminal angioplasty.

was 0.86 and 0.79 , respectively. There were no puncture site complications, such as hematoma, dissection, or pseudoaneurysm, after haemostasis with use of the previously mentioned haemostasis device in our series. 


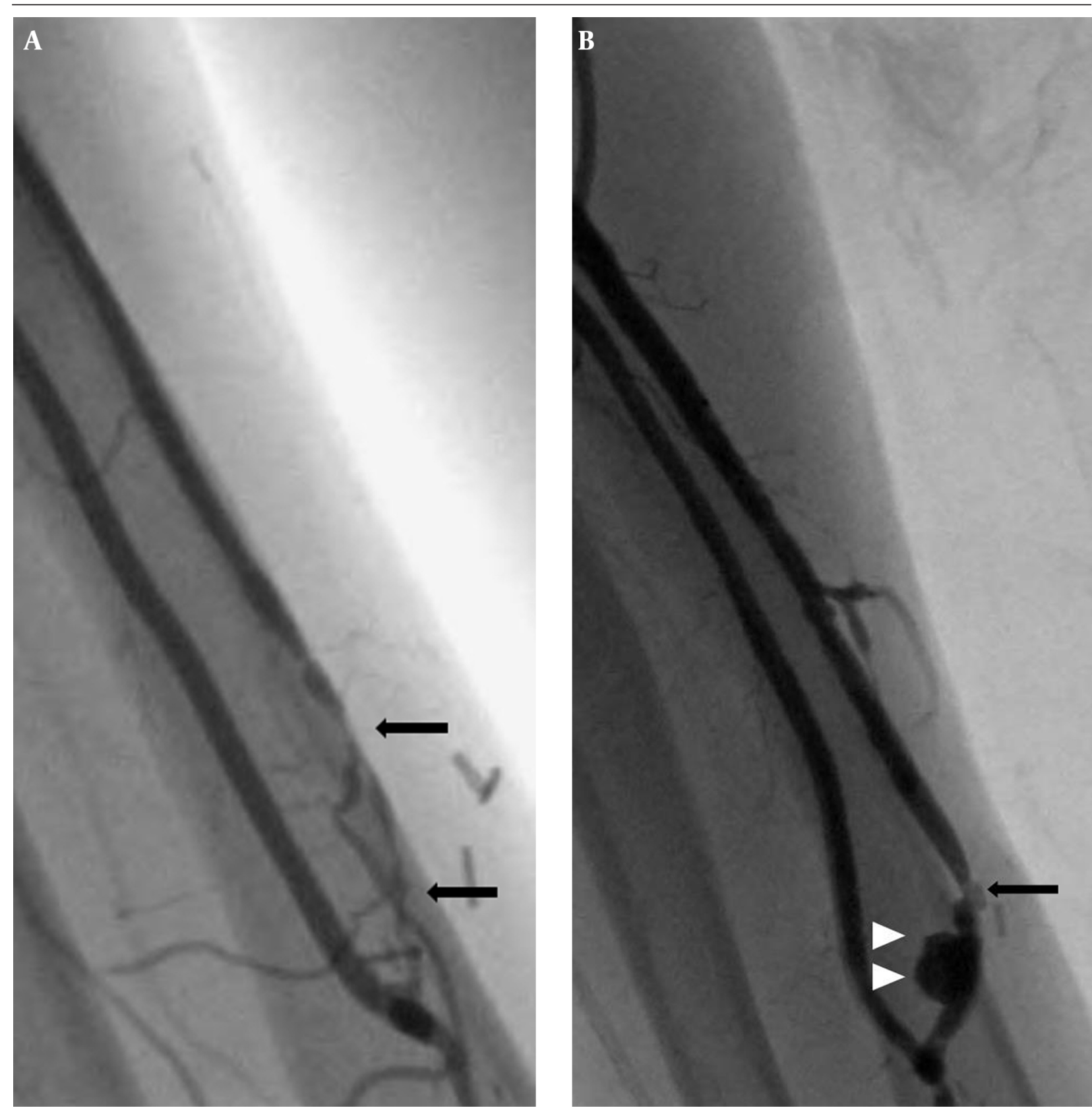

Figure 3. Failed fistula maturation after salvage PTA in a 53-year-old man with a non-maturing autogenous radiocephalic AVF; A, Initial angiography of the salvage PTA shows a severe focal stenosis about $2 \mathrm{~cm}$ in length (arrows) in the juxta-anastomotic vein of the AVF and a weak accessory vein. B, Angiography reveals an acute pseudoaneurysm (white arrowheads) that occurred immediately after balloon dilation with a $2.5 \mathrm{~mm} \times 2 \mathrm{~cm}$ balloon catheter and residual severe stenosis (arrow) in the juxta-anastomotic vein of the AVF; AVF, arteriovenous fistula; PTA, percutaneous transluminal angioplasty.

Nine patients, in whom the initial sPTA was clinically successful, had significant recurrent stenosis after haemodialysis, and nine patients underwent one additional PTA. Compared with the initial SPTA, the recurrent stenosis was observed at the same location in six patients (juxta-anastomotic vein) and at a more distal location in the trunk in three patients (venous outflow $>2 \mathrm{~cm}$ from the anastomosis). Primary patency was $96.6 \%$ at three months, $83 \%$ at six months, and $71 \%$ at 12 months.

The Cox multivariate analysis revealed that an ASR of $\geq$
0.7 (OR, 6.9; $\mathrm{P}<0.001)$ and the absence of significant residual stenosis (OR, 1.1; $\mathrm{P}<0.001)$ on the last follow-up Doppler US were significant predictors of successful aAVF maturation after SPTA. Patient factors (age; gender; co-morbidities; and age, side, and type of aAVF), vessel factors (diameter and depth of the cephalic vein on preoperative imaging, presence of accessory veins, and presence of radial artery disease), and lesion factors (type, number, and location of lesions and severity of stenosis) were not significantly associated with successful aAVF maturation (Table 4). 
Table 4. Analysis of Lesion Factors That May Predict Successful Maturation of Autogenous Arteriovenous Fistula After Salvage Percutaneous Transluminal Angioplasty ${ }^{\mathrm{a}}$

\begin{tabular}{|c|c|c|c|c|}
\hline Lesion Characteristics & No. Fistulas $(N=59)$ & Failed Maturation of AVF & Successful Maturation of AVF & P Value \\
\hline Fistula age & & & & 0.545 \\
\hline$<60$ days & 38 & 3 & 35 & \\
\hline$\geq 60$ days & 21 & 0 & 21 & \\
\hline Arterial access & & & & 0.556 \\
\hline Brachial artery & 50 & 3 & 47 & \\
\hline Radial artery & 9 & 0 & 9 & \\
\hline No. of lesions & & & & 0.536 \\
\hline Single & 45 & 1 & 44 & \\
\hline Multiple & 14 & 2 & 12 & \\
\hline Degree of stenosis & & & & 0.538 \\
\hline$\geq 90 \%$ & 13 & 2 & 11 & \\
\hline$<90 \%$ & 46 & 1 & 45 & \\
\hline Location of lesion & & & & 0.548 \\
\hline Anastomosis & 3 & 0 & 3 & \\
\hline Juxta-anastomotic vein & 47 & 3 & 44 & \\
\hline $2-10 \mathrm{~cm}$ of fistula & 5 & 0 & 5 & \\
\hline Multiple & 4 & 0 & 4 & \\
\hline Accessory vein & & & & 0.532 \\
\hline Present & 6 & 1 & 5 & \\
\hline Absent & 53 & 2 & 51 & \\
\hline Residual stenosis $>\mathbf{3 0} \%$ & & & & $<0.001$ \\
\hline Present & 3 & 3 & 0 & \\
\hline Absent & 56 & 0 & 56 & \\
\hline $\begin{array}{l}\text { Anatomic success ratio } \\
\geq 0.70\end{array}$ & & & & $<0.001$ \\
\hline Present & 56 & 0 & 56 & \\
\hline Absent & 3 & 3 & 0 & \\
\hline
\end{tabular}

\section{Discussion}

In the present study, stenosis of the cephalic vein within $5 \mathrm{~cm}$ from the arteriovenous anastomosis was the most common cause of immaturity $(84.7 \%, 50 / 59)$. Juxta-anastomotic stenosis of aAVFs has been shown to be the most common vascular lesion that causes immature aAVFs (6). Stenosis in the "venous swing point" (the region from the arteriovenous anastomosis to the point of the cephalic vein, located $5 \mathrm{~cm}$ distal to the anastomosis) is considered an acquired lesion associated with the surgical technique, as this point is the site of surgical creation and mobilization of the artery and vein (15). Other vascular lesions, such as lesions in the central veins, other venous outflow tract obstructions related to previous puncture and catheter insertion, small size of the cephalic vein, and large size of accessory veins are also well-known causes of aAVF immaturity (16).

Previous studies have identified older age, female gender, and the presence of diabetes, hypertension, and an upper-arm fistula as predictors of aAVF maturation failure. Moreover, several studies have shown that preoperative arterial and venous diameters of $>2 \mathrm{~mm}$ and 2.5 $\mathrm{mm}$, respectively, predict maturation of the aAVF (16-18). Recently, vein diameter and volume flow measured with Doppler US before and after the surgery have been suggested to be very useful for predicting fistula maturation (19). According to Robbin et al. (7), venous diameter of $>$ $4 \mathrm{~mm}$ and volume flow of $>500 \mathrm{~mL} /$ minute within four weeks after the surgery were the main determinants of successful aAVF maturation. Our attempts to evaluate immature aAVFs using the above diagnostic criteria were 
difficult because of the lack of clearly defined reference values for volume flow that would allow researchers to distinguish between mature and immature aAVFs. Furthermore, volume flow has been quantitatively studied only for matured fistulas. Therefore, appropriate cut-off values for size and volume flow need to be established for immature aAVFs (20).

Han et al. (8) demonstrated that patients aged $>65$ years and lesion factors, such as stenosis $\geq 90 \%$ and residual stenosis $\geq 20 \%$, were independently associated with reduced primary or secondary patency rate. However, Liang et al. (21) found no significant differences among three types of lesions, stenosis, obstruction, and thrombosis, on primary and secondary patency rates after SPTA. Furthermore, they found no significant difference in patency rate between groups with and without significant residual stenosis after sPTA. In agreement with the latter results, we did not find statistically significant associations between successful aAVF maturation after sPTA and various patient, vessel and lesion characteristics. Physicians should bear in mind, however, that cut-off values for the size, depth, and volume flow of the cephalic vein, associated with immature aAVFs in the Asian population, may be lower than those in the Western population, and therefore further studies are needed to verify this result.

In our study, ASR $\geq 0.7$ and the absence of significant residual stenosis were significant independent factors predicting successful fistula maturation after sPTA ( $\mathrm{P}<$ 0.001). In particular, fistulas with ASR of $\geq 0.7$ on followup Doppler US performed within two weeks after sPTA tended to have a greater rate of fistula maturation than fistulas with ASR of $<0.7$, yet this tendency was not statistically significant $(\mathrm{P}>0.05)$.

Early elastic recoil, one of the drawbacks of angioplasty, may have contributed to the above results. In this study, we performed follow-up US examinations within two weeks after SPTA to assess the diameter of the fistula. Furthermore, ASR was calculated based on the acquired maximal diameter of the venous limb of the fistula on follow-up US two weeks after SPTA and the diameter of the dilated segment of the venous limb of the fistula immediately after sPTA. This quantitative evaluation of the dilated venous limb of the fistula, during short-term follow-up Doppler US after sPTA, may be very useful for predicting successful aAVF maturation.

The appropriate size of balloon catheters for sPTA has been a matter of debate. Although same-sized balloon catheters or catheters up to $20 \%$ larger than the normal vessel diameter are often used in conventional PTA, this selection principle does not fully apply to sPTA for several reasons. First, immature fistulas in most patients from our series had very small diameters ( $\leq 3 \mathrm{~mm}$ ) $(90 \%, 53 / 59)$, which may have been smaller than the normal vessel diameter in the healthy population. Second, a larger balloon catheter increases the risk of damage to the immature vein, such as by causing a perforation or dissection. Third, immaturity typically results in a very small diameter of the distal venous limb of the fistula, and this increases the risk of vascular injury during puncture or haemostasis. Therefore, in most cases, we used same-sized balloon catheters or one approximately $0.5 \mathrm{~mm}$ smaller than the diameter of the adjacent normal venous limb of the fistula during sPTA. As a result, no major complications were observed immediately after balloon inflation except for one case of pseudoaneurysm, in which sPTA was performed for an RC-AVF with a same-sized balloon catheter ( $3 \mathrm{~mm}$ ). Even though our study had a shorter follow-up period and a smaller sample size compared to some other studies, the superior clinical success rate of this study indicates the usefulness of this approach for balloon catheter size selection for sPTA.

The rate of fistula maturation after sPTA obtained in the current study was higher than that in previous reports (6, $8)$. There may be several reasons for this difference. First, access via arterial puncture reduced the risk of damage to cephalic veins, which could preclude maturation. Second, the proportion of cases with severe stenosis (>90\%) was lower than that in other studies. Third, the incidence of meaningful accessory veins was lower than that of previous reports.

This study had several limitations that need to be addressed. First, its retrospective design may have resulted in selection or recall bias. Second, this was a single-center study, the number of patients was relatively small, and the follow-up period was relatively short, which may affect the generalizability of the results. Third, there may have been inter- or intra-observer variation in performing the Doppler US in each examination, in spite of verification of the ultrasonographic results by two board-certified interventional radiologists with $>15$ years of experience in vascular interventions. Fourth, the number of failed cases was very low in this series, which could be a major weakness of this study because the failure cases could be important in predicting the causes of aAVF maturation.

In conclusion, an ASR of $\geq 0.7$ and the absence of significant residual stenosis at the two-week follow-up Doppler US after sPTA can be used to predict successful aAVF maturation. Immediate additional sPTA was helpful for successful aAVF maturation in cases with significant residual stenosis and ASR $<0.7$.

\section{Footnotes}

Authors' Contribution:Eui-Yong Jeon and Young Kwon Cho developed the idea and played important roles in the case collection and analysis. Sung Bum Cho, Dae Young Yoon and Seong O Suh carried out the statistical analysis and reviewed the results and the discussion section of the manuscript.

Financial Disclosure:There were no conflicts of interest. Funding/Support:This study was not supported by any source from any company or any grant from any hospital or such kind of institute. 


\section{References}

1. Vascular Access Work G. Clinical practice guidelines for vascular access. Am J Kidney Dis. 2006;48 Suppl 1:S248-73. doi: 10.1053/j. ajkd.2006.04.040. [PubMed:16813991]

2. Asif A, Roy-Chaudhury P, Beathard GA. Early arteriovenous fistula failure: a logical proposal for when and how to intervene. Clin J Am Soc Nephrol. 2006;1(2):332-9. doi:10.2215/CJN.00850805. [PubMed:17699225]

3. Nassar GM. Endovascular management of the "failing to mature" arteriovenous fistula. Tech Vasc Interv Radiol. 2008;11(3):175-80. doi:10.1053/j.tvir.2008.09.004. [PubMed:19100946]

4. Persic V, Ponikvar R, Buturovic-Ponikvar J. Preoperative ultrasonographic mapping of blood vessels before arteriovenous fistula construction in elderly patients with end-stage renal disease. Ther Apher Dial. 2009;13(4):334-9. doi: 10.1111/j.17449987.2009.00735.x. [PubMed:19695070]

5. Miller GA, Hwang W, Preddie D, Khariton A, Savransky Y. Percutaneous salvage of thrombosed immature arteriovenous fistulas. Semin Dial. 2011;24(1):107-14. doi: 10.1111/j.1525-139X.2011.00846.x. [PubMed: 21338402]

6. Chung JY, Song HH, Kim KT, Kim YO, Yoon SA. Efficacy of percutaneous transluminal angioplasty for treating non-maturing Brescia-Cimino fistulas. J Korean Radiol Soc. 2004;51:281-90.

7. Robbin ML, Chamberlain NE, Lockhart ME, Gallichio MH, Young CJ, Deierhoi MH, et al. Hemodialysis arteriovenous fistula maturity: US evaluation. Radiology. 2002;225(1):59-64. doi: 10.1148/ radiol.2251011367. [PubMed:12354984]

8. Han M, Kim JD, Bae JI, Lee JH, Oh CK, Ahn C, et al. Endovascular treatment for immature autogenous arteriovenous fistula. Clin Radiol. 2013;68(6):e309-15. doi: 10.1016/j.crad.2013.01.005. [PubMed: 23465323]

9. Gray RJ, Sacks D, Martin LG, Trerotola SO. Reporting Standards for Percutaneous Interventions in Dialysis Access. Journal of Vascular and Interventional Radiology. 2003;14(9):S433-S42. doi: 10.1097/01. rvi.0000094618.61428.58. [PubMed:14514859]

10. Sidawy AN, Gray R, Besarab A, Henry M, Ascher E, Silva M, et al. Recommended standards for reports dealing with arteriovenous hemodialysis accesses. J Vasc Surg. 2002;35(3):603-10. [PubMed: 11877717]

11. Long B, Brichart N, Lermusiaux P, Turmel-Rodrigues L, Artru B, Boutin JM, et al. Management of perianastomotic stenosis of direct wrist autogenous radial-cephalic arteriovenous accesses for dialysis. J Vasc Surg. 2011;53(1):108-14. doi: 10.1016/j. jvs.2010.08.007. [PubMed: 20864300]

12. Aruny JE, Lewis CA, Cardella JF, Cole PE, Davis A, Drooz AT, et al. Quality improvement guidelines for percutaneous management of the thrombosed or dysfunctional dialysis access. J Vasc Interv Radiol. 2003;14:247-53. [PubMed:12582194]

13. Turmel-Rodrigues L, Mouton A, Birmele B, Billaux L, Ammar N, Grezard O, et al. Salvage of immature forearm fistulas for haemodialysis by interventional radiology. Nephrol Dial Transplant. 2001;16(12):2365-71. [PubMed:11733628]

14. Clark TWI, Hirsch DA, Jindal KJ, Veugelers PJ, LeBlanc J. Outcome and Prognostic Factors of Restenosis after Percutaneous Treatment of Native Hemodialysis Fistulas. J Vasc Interv Radiol. 2002;13(1):51-9. doi: 10.1016/s1051-0443(07)60009-8. [PubMed: 11788695]

15. Falk A, Teodorescu V, Lou WY, Uribarri J, Vassalotti JA. Treatment of "swing point stenoses" in hemodialysis arteriovenous fistulae. Clin Nephrol. 2003;60(1):35-41. [PubMed:12872856]

16. Zangan SM, Falk A. Optimizing arteriovenous fistula maturation Semin Intervent Radiol. 2009;26(2):144-50. doi: 10.1055/s-00291222459. [PubMed: 21326506]

17. Roy-Chaudhury P, Spergel LM, Besarab A, Asif A, Ravani P. Biology of arteriovenous fistula failure. J Nephrol. 2007;20(2):150-63. [PubMed: 17514619]

18. Silva Jr MB, Hobson Ii RW, Pappas PJ, Jamil Z, Araki CT, Goldberg $\mathrm{MC}$, et al. A strategy for increasing use of autogenous hemodialysis access procedures: Impact of preoperative noninvasive evaluation. J Vasc Surg. 1998;27(2):302-8. doi: 10.1016/s07415214(98)70360-x. [PubMed: 9510284]

19. Alamdaran A, Nazemian F, Taheri H. Doppler ultrasound assessment of well-functioning native hemodialysis access: comparison with sufficient dialysis. Iran J Radiol. 2008;5(1):101-5.

20. Kim JE, Cho YK, Sim JC, Lee SY, Sung SA, Hwang YH. Clinical Usefulness of Doppler Ultrasonography before Percutaneous Transluminal Angioplasty for Immature Native Arteriovenous Fistula. J Korean Soci Radiol. 2013;69(5):351. doi:10.3348/jksr.2013.69.5.351.

21. Liang HL, Fu JH, Wang PC, Chen MC, Wang CC, Lin YH, et al. Endovascular salvage of immature autogenous hemodialysis fistulas. Cardiovasc Intervent Radiol. 2014;37(3):671-8. doi: 10.1007/s00270014-0856-7. [PubMed: 24519641] 